\title{
Fingersight: Fingertip Visual Haptic Sensing and Control
}

\author{
George Stetten ${ }^{1,2}$, Roberta Klatzky ${ }^{3}$, Brock Nichol ${ }^{1}$, John Galeotti ${ }^{2}$, Kenneth Rockot ${ }^{1}$, \\ Kimberly Zawrotny ${ }^{1}$, David Weiser ${ }^{1}$, Nathan Sendgikoski ${ }^{1}$, Samantha Horvath ${ }^{1}$ \\ ${ }^{1}$ Department of Bioengineering, University of Pittsburgh \\ ${ }^{2}$ Robotics Institute, Carnegie Mellon University \\ ${ }^{3}$ Department of Psychology, Carnegie Mellon University
}

\begin{abstract}
We present a new method of sensing the $3 D$ visual environment and controlling objects within it. The human hand is well suited to interrogate and manipulate objects by physical contact; however, the hand is limited to surfaces within its reach. Extending the hand's innate ability, we mount miniature cameras on individual fingertips, permitting rapid sweeping through the $3 D$ visual environment at greater distances. The information gleaned from each fingertip camera is fed back to that finger by a small vibrator, so the sense of touch remains related to each finger's individual interaction with the environment. The muscles of the fingers, wrist, and arm can potentially provide motor control as fine as the eyes' (and we have ten fingers instead of only two eyes) although the sensory resolution and total bandwidth is likely to be less. Metaphorically speaking, we have given eyesight to the fingers, and thus we call the resulting capability, "Fingersight ${ }^{T M}$." In addition to sensing the environment, we can remotely control certain identified targets with subsequent motion of the fingers. We can move or rotate objects on a computer screen or recognize inanimate objects such as a light switch, controlling environmental parameters with a flick of the finger.
\end{abstract}

Keywords - sensory aids, tactile sensors, machine vision, image analysis.

\section{INTRODUCTION}

Many devices that transfer input from the visual environment to another sense have been developed. For example, visually impaired individuals have been presented with a wide variety of devices that transfer to another sense at least some of the vision-related capabilities of normally sighted individuals. The primary assistive technologies currently used by the blind to navigate through the environment are essentially unchanged from those used twenty years ago, namely, white canes and guide dogs. [1] Although these two methods can facilitate the ability to travel safely in a wide variety of environments, neither provide the kind of assistance needed to straighten a picture frame on the wall or find a can of soup on a counter-top.

Electronic navigation aids are finding some acceptance, especially laser and ultrasound canes, as well as portable computers with global positioning systems (GPS) and electronic Braille or speech interfaces. [2] Replacing the more general capabilities of vision to provide detailed information about objects in the environment has proven more difficult.
Existing systems capture an image and then display that image on a tactile screen that can be worn on a belt, for example. The fingers can then be used to interrogate the image depicted on the tactile screen in an effort to "visualize" the image depicted on the screen. [3,4] Performing what Bach-y-Rita calls Tactile-Vision Sensory Substitution (TVSS), such devices have also been developed to directly stimulate the visual cortex and the tongue. [5] Others, such as the Optacon (Telesensory Corp., defunct), have been developed specifically for reading the printed page. [6] These all preserve a predetermined map between the image captured by a camera and a spatially fixed grid of sensory stimulators.

There are also a number of related technologies that monitor hand, eye, and body motion from a fixed camera or sensor array to interpret gestures, commands, gaze direction, e.g. [7]. These are generally not worn by the operator and thus only operate within a limited space, focusing on the motions of the operator hmself rather than his interrogation of whatever environment he happens to be in.

A number of devices have been developed to communicate haptic feedback to the hand from virtual environments, such as the CyberTouch ${ }^{\mathrm{TM}}$ Glove (Immersion Corp., San Jose, CA), which uses optical tracking of each finger to determine when vibratory stimulators on that finger should be activated. These devices aim to simulate tactile exploration of virtual objects.

There exists a need, however, for a device that allows active interrogation and sensing of the actual 3D visual environment surrounding the operator while moving through daily life. There also exists a need for a device that not only allows the user to sense the environment but also provides control of specific aspects of the environment that are sensed by the device. These abilities to interrogate and control the environment should not necessarily be limited to a specific, predetermined environment.

Our original goal was to serve the population who are visually impaired, and to do so we looked for a sensory channel to substitute for vision. The main candidates for such a substitute sensory channel are touch and hearing. Although hearing offers a potentially higher bandwidth, it is crucial not to impede its existing use, which can be acutely well developed by those who lack normal vision, providing essential acoustic cues about the environment. [8] As for touch, the hands offer the greatest versatility and sensitivity, 
but still we must not completely usurp their use, since they are essential for so many tasks in daily living.

We introduce here a concept we call Fingersight ${ }^{\mathrm{TM}}$, which may be embodied in a number of ways to provide a variety of different sensing and control devices for use as surrogates for vision and direct manipulation. Since the implementations presented here represent a very early stage of development, this paper is meant simply to explain the underlying concept and explore possible directions for its evolution.

There are two major components to the concept, relating respectively to sensing and controlling the environment. These are addressed sequentially in the following two sections. As already mentioned, the sensory component was originally designed to assist the visually impaired. The introduction of control into Fingersight has expanded the potential target population to the general public, who could make use of it as an intuitive and possibly even enjoyable new form of remote control.

\section{FINGERTIP SENSING OF THE VISUAL ENVIRONMENT}

The sensing portion of Fingersight involves perceiving the world in a fundamentally new way. Each finger interrogates an individual region of the visual environment, and may identify some particular feature or object. Each finger has, in the parlance of visual perception, its own receptive field. Motion of the finger, hand, and arm replace motion of the eye and head in eyesight, controlling that finger's individual field of view. A miniature video camera, mounted on the dorsal aspect (top or nail side) of a particular finger provides an image for real time analysis, which is used to identify the particular feature or object. This may be an elementary feature as basic as a properly oriented edge, or an object as complex as a particular person's face. We use the dorsal aspect of the finger to mount the camera so as to minimize interference with normal use of the hand. The output of the image analysis is used to control haptic stimulation of that finger, so that each finger "feels" what has been identified in the receptive field of that finger.

We implemented a previous version of Fingersight before the recent advent of inexpensive miniature cameras. [9] As shown in Figure 1, it uses a small laser attached to the finger and suspended by a spring in such a way that the laser can be made to vibrate vertically by a solenoid (consisting of an electromagnetic coil and small permanent magnets). Light from the laser (modulated at $40 \mathrm{KHz}$ ) is reflected from objects in the environment and detected by a phototransistor. The strength of the reflection is determined by the distance, reflectance, orientation, and color of the object. By amplifying the received signal and using it to power the solenoid, and thus to change the orientation of the laser, it is possible to create regenerative feedback, much in the same way that a microphone will "feed back" when placed too close to the speaker of an audio amplifier.

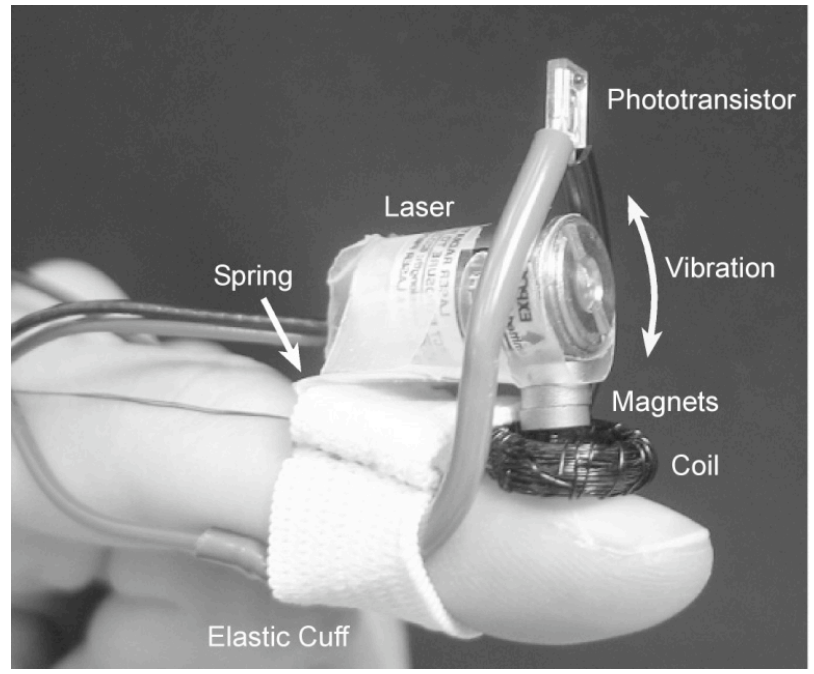

Fig.1. Fingertip-mounted vibrating laser for sensing visual edges through regenerative feedback.

Moving the laser across an optical edge (e.g., at the boundary of an object or an abrupt change in its color) causes a change in the amount of reflected laser light. This change, when amplified and fed back to the solenoid, results in oscillatory motion of the laser. This oscillation is felt by the fingertip when a properly oriented edge has been located in the visual environment. The use of several such devices on different fingers providing oriented edge detection could permit the ability to discriminate a table top from a doorway, or the edge of a sidewalk, for example.

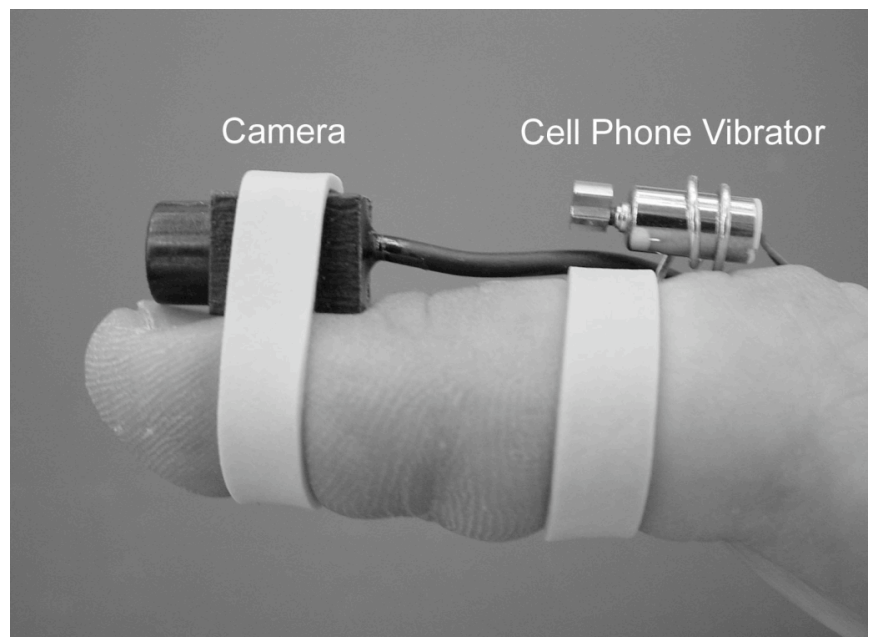

Fig. 2. Experimental prototype showing camera mounted on distal phalanx and cell phone vibrator mounted on medial phalanx of the index finger.

More recently, we have attached a very small and inexpensive video camera (made available by the cell phone industry) to the finger as shown in Fig. 2. The advantage of the camera is that we can identify not just edges, but also a wide variety of features and objects, using one of many algorithms available from the field of computer vision. Thus 
the operator could "feel" at a distance a particular doorknob or coffee mug, or even identify a particular person in a crowd. Multiple fingers could quickly locate a number of different objects or people with a sweep of the hand. Our present model uses a miniature cell phone vibrator (Fig. 2) to provide tactile stimulation. The vibrator is attached more proximally on the finger to avoid undue disturbance of the video image.

\section{FINGERTIP CONTROL OF IDENTIFIED TARGETS}

With the evolution of Fingersight came an additional idea that once a target has been located, control of the environment could be accomplished by subsequent motion of the finger. Thus, a light switch could be identified in the camera image, as communicated to the operator by vibration of the finger, and subsequent vertical motion of the finger (detected by image motion) could then turn the light on and off. We have implemented a number of such systems to demonstrate the concept. In the first (see Fig. 3), the location of a small white square on a screen is controlled by motion of the finger. The algorithm detects the square in the field of view of the camera, moving it on the screen to keep it constantly in the center of the camera image, and providing haptic feedback whenever the tracking system has locked onto the target. Except for the haptic feedback to the individual fingertip, this is similar to a system described by Silber. [10]

A variation on this system constrains the square to move along a straight line, simulating the action of a slide pot under the operator's remote control. A further variation uses a small white triangle to simulate a knob, whose orientation is determined using standard computer vision techniques and subsequently controlled by rotation of the finger.

\section{FUTURE DIRECTIONS}

Clearly, such systems are not limited to actively controlled graphical objects on a screen, but could identify inanimate objects such as the abovementioned light switch or doorknob. In such cases, remote control could still be achieved by motion of the finger once the target had been identified, using a separate control channel to turn on the light or open the door.

Furthermore, by analyzing the foreshortening and scale of certain objects, it should be possible to determine one's location relative to them. Thus particular graphical shapes mounted at known locations could provide the user with navigational cues. For example, a specially designed geometrical figure attached to the refrigerator door could provide the operator with information regarding his relative distance and orientation to the refrigerator.

For haptic stimulation, we are currently using miniature vibrators commonly used in cell phones, which operate by having a small motor spin an off-axis weight. This design is more efficient at producing vibration than miniature speakers, which do not store significant energy in their reciprocating motion. However, cell phone vibrators do not allow independent control of amplitude and frequency, since a single DC voltage controls both parameters. Many other forms of haptic stimulation could be used, and this is an active field of development by many researchers. The prospect of multiple channels of information to each finger broadens the types of image analysis that could be used in our system.

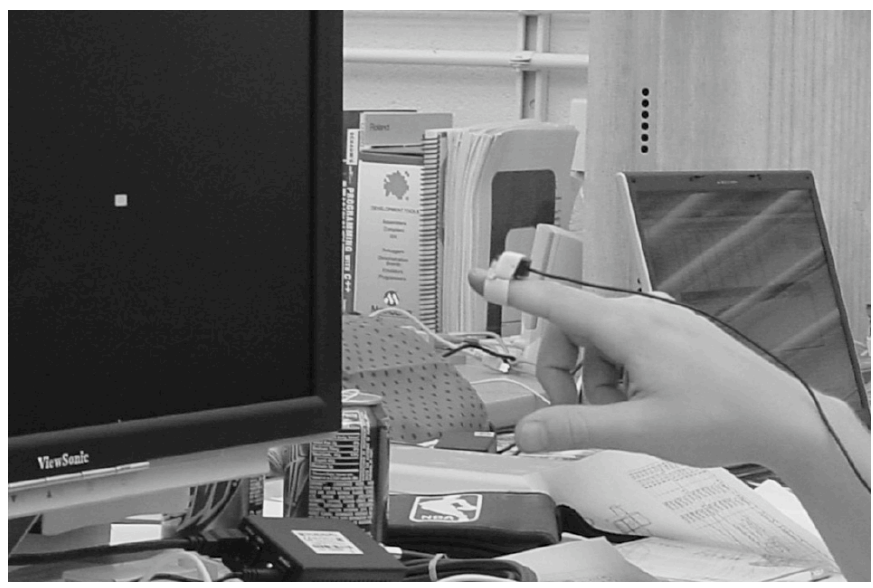

Fig.3. Fingertip-mounted camera controlling the location of a small square target on a screen (miniature vibrator not shown).

We are just at the very beginning stages of exploring the possibilities of Fingersight. As opposed to previous methods of visual-tactile substitution, our system does not depend upon a fixed spatial map between the image and the sensory stimulators. Rather, we use each individual finger to explore what amounts to its own receptive field in the visual environment. Besides offering a potentially useful tool for the visually impaired, Fingersight presents an intriguing apparatus for the general public, potentially well suited to the entertainment industry. It also opens up a new realm for psychophysical study of the use of touch in the sensing and control of the visual environment. Human vision offers a rich trove of abilities that may be transferable to this new method of sensing the visual environment.

We have submitted a patent application on Fingersight.

\section{ACKNOWLEDGEMENTS}

NSF Robotics and Human Augmentation Grant no. 0308096

\section{REFERENCES}

[1] E. R. Strelow, and D. H. Warren, (Ed.), Electronic Spatial Sensing for the Blind, Dordrecht: Martinus Nijhoff Publishers, 1985.

[2] J. L. Loomis, R. Golledge, R.L. Klatzky, and J. Marston, (in press) "Assisting Wayfinding in Visually Impaired Travelers." In G. Allen (Ed.), Applied Spatial Cognition: From Research to Cognitive Technology, Mahwah, N.J.: Lawrence Erlbaum Associates

[3] R. Velázquez, E. Pissaloux, M. Wiertlewski, "A Compact Tactile Display for the Blind with Shape Memory Alloys," International Conference on Robotics and Automation, 2006. 
[4] F. Maingreaud, E. Pissaloux, F. Gaunet, R. Velazquez, M. Hafez, J-M. Alexandre, "A dynamic tactile map as a tool for space organization perception: application to the design of an electronic travel aid for visually impaired and blind people," 27th Annual Int. Conf. of the IEEE Engineering in Medicine and Biology Society, Shanghai, China, Sept. $1-4,2005$.

[5] P. Bach-y-Rita, M.E. Tyler, K.A. Kaczmarek, "Seeing with the brain," International journal of human-computer interaction 2003 (15)2:285295.

[6] J. C. Craig, "Vibrotactile pattern perception: extraordinary observers," Science. 1977; 196(4288):450-2.

[7] J. Sibert, M. Gokturk, "Direct pointing apparatus and method therefor," US Patent 6184863, Feb 6, 2001.

[8] R. G., Golledge, J. Loomis, R. L., Klatzky, and J. R. Marston, "Stated Preferences for Components of a Personal Guidance System for Nonvisual Navigation", Journal of Visual Impairment and Blindness, 98, 135-147, 2004.

[9] K. Zawrotny, A. Craig, D. Weiser, R. Klatzky, and G. Stetten, "Fingertip Vibratory Transducer for Detecting Optical Edges using Regenerative Feedback," Haptic Interfaces for Virtual Environment and Teleoperator Systems, IEEE Virtual Reality 2006, pp. 373-374, Arlington VA, March 2006.

[10] M. Silber, "Using video information to control cursor position," US Patent 6,738,041, May 18, 2004. 